\title{
Entropy of the QCD Plasma
}

\author{
J.-P. Blaizot \\ Service de Physique Théorique, CE Saclay, F-91191 Gif-sur-Yvette, France \\ E. Iancu \\ Theory Division, CERN, CH-1211 Geneva 23, Switzerland
}

\begin{abstract}
A. Rebhan
Institut für Theoretische Physik, Technische Universität Wien, Wiedner Hauptstraße 8-10/136, A-1040 Vienna, Austria
\end{abstract}

(Received 11 June 1999)

\begin{abstract}
Self-consistent approximations in terms of fully dressed propagators provide a simple expression for the entropy of an ultrarelativistic plasma, which isolates the contribution of the elementary excitations as a leading contribution. Further approximations, whose validity is checked on a soluble model involving a scalar field, allow us to calculate the entropy of the QCD plasma. We obtain an accurate description of lattice data for purely gluonic QCD, down to temperatures of about twice the transition temperature.
\end{abstract}

PACS numbers: 12.38.Mh, 11.10.Wx

The properties of the high temperature phase of QCD cannot be easily calculated using perturbation theory, in spite of the fact that the gauge coupling $g$ is small if the temperature $T$ is sufficiently high. This is evidenced, in particular, by the poor convergence properties of the perturbative series [1].

Lattice results, which show that the ideal gas limit is approached as $T$ becomes large, can be accounted for reasonably well by phenomenological fits involving massive quasiparticles [2,3]. Although the quasiparticle picture suggested by such fits is a rather crude representation of the actual physics of non-Abelian gauge theories, it supports the idea that one should be able to give an accurate description of the thermodynamics of the QCD plasma in terms of its elementary excitations.

It is worth emphasizing at this stage that, among the relevant degrees of freedom, the soft collective ones, with momenta of order $g T$, are clearly nonperturbative. Although their leading order contribution $\propto g^{3}$ to the pressure can be easily isolated [4], it does not make much physical sense to regard this contribution as a genuine perturbative correction. Recent investigations [5-7] indicate indeed that trying to represent this contribution by a truncated polynomial in $g$ is not appropriate.

In order to carry out a more complete calculation, we shall use techniques which allow systematic rearrangements of the perturbative expansion, avoiding double countings. We shall rely, in particular, on self-consistent approximations which provide a simple expression for the entropy, isolating the contribution of the elementary excitations as a leading contribution. We show that this entropy formula can be used to get a good estimate of the QCD entropy at high temperature. The results that we have obtained so far are quite encouraging and give us the hope that an analytical control of the high temperature phase of QCD is within reach.
We shall first discuss scalar field theories with $g \varphi^{3}+$ $g^{2} \varphi^{4}$ interactions, with the double purpose of presenting the general framework and of checking approximations which will be used later for QCD.

The thermodynamic potential $\Omega=-P V$ of the scalar field can be written as the following functional of the full propagator $D[8,9]$ :

$$
\beta \Omega[D]=-\log Z=\frac{1}{2} \operatorname{Tr} \log D^{-1}-\frac{1}{2} \operatorname{Tr} \Pi D+\Phi[D],
$$

where $\operatorname{Tr}$ denotes the trace in configuration space, $\beta=$ $1 / T$, and $\Phi[D]$ is the sum of the two-particle-irreducible "skeleton" diagrams

$$
-\Phi[D]={ }_{1 / 12} \bigcirc+1 / 8 \bigcirc+1 / 48 \bigcirc+\ldots .
$$

The self energy $\Pi=D^{-1}-D_{0}^{-1}$, where $D_{0}$ is the bare propagator, is related to $\Phi[D]$ by

$$
\delta \Phi[D] / \delta D=\frac{1}{2} \Pi .
$$

An important property of the functional $\Omega[D]$, which is easily verified using (3), is that it is stationary under variations of $D$,

$$
\delta \Omega[D] / \delta D=0 .
$$

Self-consistent (“ $\Phi$-derivable”) [9] approximations, i.e., approximations which preserve this property, are obtained by selecting a class of skeletons in $\Phi[D]$ and calculating $\Pi$ from Eq. (3) above.

The stationarity of $\Omega[D]$ has an interesting consequence for the entropy. Because of Eq. (4) the temperature derivative of the spectral density in the dressed propagator cancels out in the entropy density

$$
S=-\partial(\Omega / V) / \partial T
$$


and one obtains $[10,11]$

$$
\begin{aligned}
S= & -\int \frac{d^{4} k}{(2 \pi)^{4}} \frac{\partial n(\omega)}{\partial T} \operatorname{Im} \log D^{-1}(\omega, k) \\
& +\int \frac{d^{4} k}{(2 \pi)^{4}} \frac{\partial n(\omega)}{\partial T} \operatorname{Im} \Pi(\omega, k) \operatorname{Re} D(\omega, k)+S^{\prime}
\end{aligned}
$$

with

$$
S^{\prime}=-\left.\frac{\partial(T \Phi)}{\partial T}\right|_{D}+\int \frac{d^{4} k}{(2 \pi)^{4}} \frac{\partial n(\omega)}{\partial T} \operatorname{Re} \Pi \operatorname{Im} D=0
$$

up to terms that are of loop-order 3 or higher. Thus, in contrast to $\Omega$, where $\Phi$ contributes already to order $g^{2}$ in perturbation theory, Eq. (6) with $S^{\prime}=0$ is perturbatively correct up to, and including, order $g^{3}$ [12]. The first two terms in Eq. (6) represent essentially the entropy of "independent quasiparticles," while $S^{\prime}$ may be viewed as the residual interactions among these quasiparticles [11].

Besides this important simplification, $S$, in contrast to the pressure, has the advantage of manifest ultraviolet finiteness, since $\partial n / \partial T$ vanishes exponentially for both $\omega \rightarrow \pm \infty$. Moreover, any multiplicative renormalization $D \rightarrow Z D, \Pi \rightarrow Z^{-1} \Pi$ with real $Z$ drops out from Eq. (6).

We now focus on the self-consistent approximation obtained in $g^{2} \varphi^{4}$ theory where only the second diagram in Eq. (2) for $\Phi$ is kept. Then $\operatorname{Im} \Pi=0$ and $\operatorname{Re} \Pi=$ $m^{2}=$ const, and Eq. (6) reduces to

$$
\begin{aligned}
S & =-\int \frac{d^{4} k}{(2 \pi)^{4}} \frac{\partial n(\omega)}{\partial T} \operatorname{Im} \log \left(k^{2}-\omega^{2}+m^{2}\right) \\
& =\frac{4}{T}\left[\frac{\pi^{2} T^{4}}{90}-\frac{m^{2} T^{2}}{48}+\frac{m^{3} T}{48 \pi}+\ldots\right] .
\end{aligned}
$$

A fully self-consistent determination of $m$ corresponds to solving the gap equation

$$
m^{2}=12 g^{2} \int \frac{d^{4} k}{(2 \pi)^{3}} n(\omega) \epsilon(\omega) \delta\left(\omega^{2}-k^{2}-m^{2}\right) .
$$

When the solution of this equation is inserted in Eq. (8), the entropy obtained coincides with that of the exact solution of a scalar $\mathrm{O}(N)$ model in the limit of $N \rightarrow \infty[7,13]$. Note that in contrast to Eq. (8), the gap equation is ultraviolet divergent (for $\omega \rightarrow-\infty$ ), and requires renormalization [7], affecting $m^{2}$ at perturbative order $g^{4}$ and beyond.

In view of the subsequent application to QCD, where a fully self-consistent determination of the gluonic self-energy seems prohibitively difficult, we consider now perturbative approximations. Our goal is to obtain approximate expressions for the self-energy which allow us to reproduce the perturbative result for the entropy when expanded to order $g^{3}$. We emphasize that our final results for the entropy are nonperturbative, and not limited to a truncated polynomial in $g$. What we are testing here is the quality of approximations which preserve self-consistency up to order $g^{3}$ at least, which is what we shall be able to do in QCD.
As first approximation we consider the leading contribution to the self-energy at high temperature, the so-called hard thermal loop (HTL) [14], and refer to Eq. (6) with this restriction as $S_{\mathrm{HTL}}$. For the $\varphi^{4}$ theory, we have simply $\Pi \rightarrow \hat{\Pi}=\hat{m}^{2}=g^{2} T^{2}$. When inserted into Eq. (8), this yields the correct result for the leading-order interaction term $g^{2} T^{3}$ in the entropy.

On the other hand, the order $g^{3}$ contribution contained in $S_{\mathrm{HTL}}$ turns out to be too small by a factor of 4 when compared to the well-known perturbative result [4]. This is corrected by including the next-to-leading order (NLO) term in the thermal mass through resummed perturbation theory, $m^{2}=\hat{m}^{2}+\delta m^{2}=g^{2} T^{2}-\frac{3}{\pi} g^{3} T^{2}$ [4]. So, when compared to conventional resummed perturbation theory, the order- $g^{3}$ term arises in an unusual manner: whereas in the former the entire plasmon effect comes from the infrared regime, in Eq. (6) an even larger contribution comes indirectly from the infrared through corrections to the dispersion laws relevant at hard momenta [the $T^{2}$ term in Eq. (8) comes entirely from hard $k \sim T$ ]. This may be understood as a consequence of the requirement of self-consistency: recall that Eq. (8) relies on the stationarity of the thermodynamic potential, and this has to be maintained at the order of interest.

At large coupling the NLO result for $m^{2}$ inevitably turns negative. This can be avoided by taking instead the perturbatively equivalent form $\hat{m}^{2}+\delta m^{2}=g^{2} T^{2} /[1+$ $3 g / \pi$, which is monotonous in $g$, giving a very good approximation to the solutions of Eq. (9) up to $g \geqslant 1$.

In Fig. 1 we compare the various approximations numerically with the exact entropy (full line), normalized to their ideal-gas values (SB), as functions of the renormalized coupling in the modified minimal subtraction ( $\overline{\mathrm{MS}}$ ) scheme. In contrast to the full result, the perturbative approximations are renormalization-scale dependent. As in Ref. [7] we consider the effect of varying the scale $\bar{\mu}$, here in the range $\bar{\mu}=\left(\frac{1}{2}-2\right) \times 2 \pi T$. The lower and upper dark-gray bands correspond to conventional perturbative results for $S / S_{\mathrm{SB}}$ up to order $g^{2}$ and $g^{3}$, respectively, the medium-gray bands to the result of HTL and NLO resummations of the two-loop entropy. The latter, which clearly

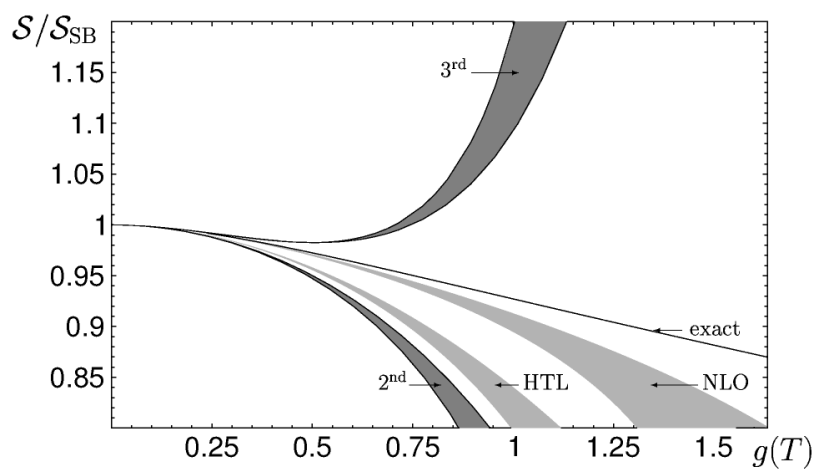

FIG. 1. Comparison of perturbative and HTL-improved approximations to the entropy in the large- $N$ scalar $\mathrm{O}(N)$ model. See text for detailed explanations. 
represent a substantial improvement over the former, are the approximations that we shall now implement for QCD.
The analog of Eq. (6) in purely gluonic QCD and in Coulomb gauge reads

$$
\begin{gathered}
S=-N_{g} \int \frac{d^{4} k}{(2 \pi)^{4}} \frac{\partial n(\omega)}{\partial T}\left\{2 \operatorname{Im} \log \left(-\omega^{2}+k^{2}+\Pi_{T}\right)+2 \operatorname{Im} \Pi_{T} \operatorname{Re}\left[\omega^{2}-k^{2}-\Pi_{T}\right]^{-1}\right. \\
\left.+\operatorname{Im} \log \left(k^{2}+\Pi_{L}\right)-\operatorname{Im} \Pi_{L} \operatorname{Re}\left[k^{2}+\Pi_{L}\right]^{-1}\right\}
\end{gathered}
$$

with $N_{g}=N^{2}-1=8$ for SU(3). The (spatially) transverse $\left(\Pi_{T}\right)$ and longitudinal $\left(\Pi_{L}\right)$ structure functions will be specified below. Here we have assumed that the gluon self-energy is transverse with respect to the fourmomentum, and that there are no contributions from the ghosts, which turns out to be justified in the approximations that we shall be interested in [12].

The order $g^{2}$ contribution to the entropy is easily extracted from Eq. (10),

$$
\begin{aligned}
S^{(2)}= & -2 \pi N_{g} \int \frac{d^{4} k}{(2 \pi)^{4}} \frac{\partial n}{\partial T} \\
& \times \epsilon(\omega) \delta\left(\omega^{2}-k^{2}\right) \operatorname{Re} \Pi_{T}(\omega, k) \\
= & -N_{g} \frac{m_{\infty}^{2} T}{6}=-\frac{N N_{g}}{36} g^{2} T^{3} .
\end{aligned}
$$

Here we have used the fact that the integral is dominated by hard momenta and that the transverse quasiparticles have the asymptotic thermal mass,

$$
m_{\infty}^{2}=\Pi_{T}\left(\omega^{2}=k^{2}\right)=g^{2} N T^{2} / 6 .
$$

This latter result is exact at the bare one-loop level [15].

The contribution of order $g^{3}$ involves loop integrals with soft momenta, which requires using the HTL approximation for $\Pi$, where $[14,16]$

$$
\begin{gathered}
\hat{\Pi}_{L}(\omega, k)=\hat{m}_{D}^{2}\left[1-\frac{\omega}{2 k} \log \frac{\omega+k}{\omega-k}\right], \\
\hat{\Pi}_{T}(\omega, k)=\frac{1}{2}\left[\hat{m}_{D}^{2}+\left(\frac{\omega^{2}}{k^{2}}-1\right) \hat{\Pi}_{L}\right],
\end{gathered}
$$

with $\hat{m}_{D}=g T \sqrt{N / 3}$. The spectral density of the corresponding gluon propagator consists of quasiparticle poles with momentum-dependent effective masses and Landau damping cuts for $|\omega|<k$. When $k \gg g T$, the additional pole associated to the collective longitudinal excitation has exponentially vanishing residue [17].

The order $-g^{3}$ contribution in $S_{\mathrm{HTL}}$ can be isolated as

$$
S_{\mathrm{HTL}}^{(3)} / N_{g}=\int \frac{d^{4} k}{(2 \pi)^{4}} \frac{1}{\omega}\left\{2 \operatorname{Re} \hat{\Pi}_{T}\left[\operatorname{Im}\left(\omega^{2}-k^{2}-\hat{\Pi}_{T}\right)^{-1}-\operatorname{Im}\left(\omega^{2}-k^{2}\right)^{-1}\right]-\operatorname{Re} \hat{\Pi}_{L} \operatorname{Im}\left(k^{2}+\hat{\Pi}_{L}\right)^{-1}\right\}-\frac{\hat{m}_{D}^{3}}{24 \pi} \text {. }
$$

For the same reason as in the above scalar example, this is only part of the full order $g^{3}$ contribution. Remarkably, it turns out that, as in the scalar case, Eq. (15) is precisely $1 / 4$ of the correct result $S^{(3)}=N_{g} \hat{m}_{D}^{3} /(3 \pi)$.

The remaining order- $g^{3}$ correction comes from Eq. (11) with $\delta \Pi_{T}$ in place of $\hat{\Pi}_{T}$, with $\delta \Pi_{\mu \nu}$ being evaluated at order $g \hat{m}_{D}^{2}$ in HTL-resummed perturbation theory. The expression (11) is dominated by hard momenta $k \sim T$, and to the order of interest $\delta \Pi_{T}$ is given by the two contributions shown in Fig. 2, in which one internal line is hard, and the other one is a soft resummed longitudinal (a) or transverse (b) gluon propagator. Diagram 2(a) restores the correct combinatorial factor of the longitudinal ring diagrams, whereas diagram 2(b) compensates for spurious transverse plasmon effects that are present in the HTL approximation $S_{\mathrm{HTL}}[12]$.

We turn now to the numerical evaluation of $S_{\mathrm{HTL}}$ and shall discuss the effects of the above NLO contributions

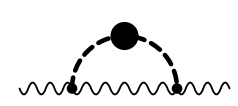

(a)

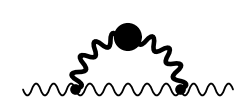

(b)
FIG. 2. NLO contributions to $\delta \Pi_{T}$ at hard momentum. Thick dashed and wiggly lines with a blob represent HTL-resummed longitudinal and transverse propagators. further below. $S_{\mathrm{HTL}}$ involves two physically distinct contributions. One corresponds to the transverse and longitudinal gluonic quasiparticle poles,

$$
\begin{aligned}
S_{\mathrm{HTL}}^{\mathrm{QP}}=-N_{g} \int \frac{d^{3} k}{(2 \pi)^{3}} \frac{\partial}{\partial T}[ & 2 T \log \left(1-e^{-\omega_{T}(k) / T}\right) \\
& \left.+T \log \frac{1-e^{-\omega_{L}(k) / T}}{1-e^{-k / T}}\right],
\end{aligned}
$$

where only the explicit $T$ dependences are to be differentiated, and not those implicit in the HTL dispersion laws $\omega_{T}(k)$ and $\omega_{L}(k)$. The latter are given by the solutions of $\omega_{T}^{2}-k^{2}=\hat{\Pi}_{T}\left(\omega_{T}, k\right)$ and $k^{2}=-\hat{\Pi}_{L}\left(\omega_{L}, k\right)$ [16].

Secondly, there are the Landau-damping contributions which read

$$
\begin{aligned}
S_{\mathrm{HTL}}^{\mathrm{LD}}=- & N_{g} \int_{0}^{\infty} \frac{k^{2} d k}{2 \pi^{3}} \int_{0}^{k} d \omega \frac{\partial n(\omega)}{\partial T} \\
\times & {\left[2 \arg \left(k^{2}-\omega^{2}+\hat{\Pi}_{T}\right)\right.} \\
& +2 \operatorname{Im} \hat{\Pi}_{T} \operatorname{Re}\left(\omega^{2}-k^{2}-\hat{\Pi}_{T}\right)^{-1} \\
& \left.+\arg \left(k^{2}+\hat{\Pi}_{L}\right)-\operatorname{Im} \hat{\Pi}_{L} \operatorname{Re}\left(k^{2}+\hat{\Pi}_{L}\right)^{-1}\right] .
\end{aligned}
$$

The usual perturbative $g^{2}$ contribution (11) is contained in the first term of Eq. (16); all the other terms in Eqs. (16) and (17) are of order $g^{3}$ in a small-g expansion. 


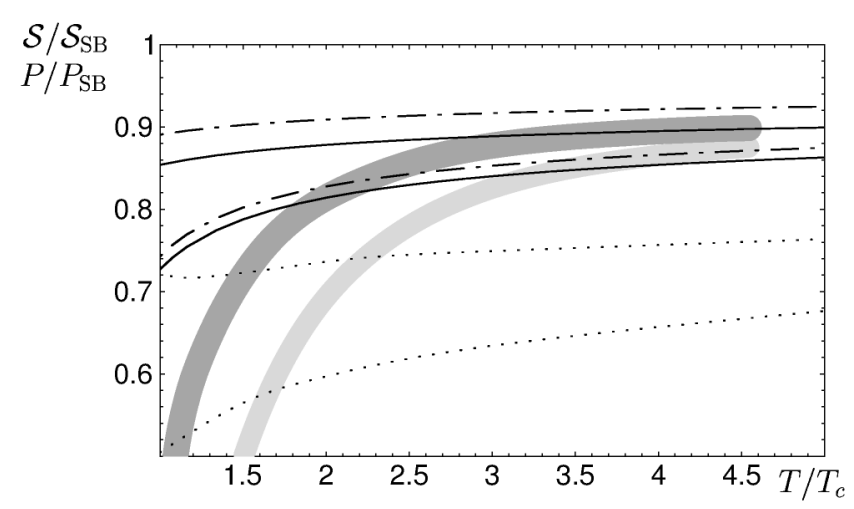

FIG. 3. HTL-improved results for the two-loop entropy $S / S_{\mathrm{SB}}$ in purely gluonic QCD (full lines) with $\bar{\mu}$ varied between $\pi T$ and $4 \pi T$; our estimates for NLO effects are given by the dash-dotted lines. The lattice result for the entropy is represented by the dark-gray band. For comparison, the HTL-resummed results of Ref. [19] for the one-loop pressure are given by the dotted lines, the lattice results for $P / P_{\mathrm{SB}}$ by the light-gray band. (Because of the weak temperature dependence of the theoretical result the prediction of Ref. [19] for $P / P_{\mathrm{SB}}$ is approximately that for $S / S_{\mathrm{SB}}$.)

In Fig. 3, we compare the numerical evaluation of $S_{\mathrm{HTL}} / S_{\mathrm{SB}}$ with the lattice data for purely gluonic QCD from Ref. [18], in the same manner as done in Ref. [19], i.e., we use the two-loop running coupling constant $\alpha_{s}(\bar{\mu})$ of the $\overline{\mathrm{MS}}$ scheme with $\Lambda_{\overline{\mathrm{MS}}}=T_{c} / 1.03$ and the renormalization scale is varied in the range $\bar{\mu}=\left(\frac{1}{2}-2\right) \times$ $2 \pi T$ to give an estimate of the theoretical uncertainty.

The thick dark-gray line represents the lattice data for the entropy density with the thickness of the line giving roughly the error reported in Ref. [18]. Our result reproduces the lattice data rather well already for $T \geq 2 T_{c}$.

Also given in the same figure are the lattice data for the pressure $P / P_{\mathrm{SB}}$ (the lower light-gray band) and the result for the full HTL resummation of the one-loop pressure reported in Ref. [19] (dotted lines). The discrepancy between the latter can be attributed in part to an overcounting in the interaction pressure in Ref. [19], which will be corrected only in a fully resummed two-loop calculation. By contrast, our approach has the advantage of including the correct leading order interaction terms already in a pure HTL approximation, and also of manifest ultraviolet finiteness, thus avoiding the introduction of artificial counterterms depending on the thermal mass.

Turning now to the NLO approximation to $S$, we note that, at hard momenta $k, \operatorname{Re} \delta \Pi_{T}\left(\omega^{2}=k^{2}\right)$ is, unlike $m_{\infty}^{2}$, a nonlocal quantity. In order to get some estimate on its numerical effect, we approximate it by a constant correction which has the right magnitude to produce the known perturbative coefficient of order $g^{3}$. We choose this as $m_{\infty}^{2}+\delta m_{\infty}^{2}=g^{2} T^{2} N /[6(1+\sqrt{3 N} g / \pi)]$, and include this correction only in the hard momentum region defined by $k>M=\sqrt{2 \pi T m_{D}}$. The reason for this is that we do not want to change by hand the overall scale of HTL contributions in the soft regime, where NLO contributions are known to behave quite differently: the long-wavelength plasma frequency receives much smaller negative corrections [20], and the Debye screening mass is known to be even substantially increased [21,22].

The dash-dotted lines in Fig. 3 give the correspondingly modified numerical results. In addition to a variation of $\bar{\mu}$ we have also included a variation of $M$, the boundary between hard and soft momenta, by a factor of 2 around its central value. These results happen to describe the lattice data surprisingly well, although their primary significance is to demonstrate the relative stability of our scheme upon the inclusion of terms that restores equivalence with the known perturbative result up to and including order $g^{3}$. A full NLO calculation still remains to be done and will be presented in a forthcoming publication.

This work was supported by the Austrian-French scientific exchange program Amadeus of APAPE and ÖAD.

[1] P. Arnold and C. Zhai, Phys. Rev. D 51, 1906 (1995); E. Braaten and A. Nieto, Phys. Rev. D 53, 3421 (1996).

[2] A. Peshier, B. Kämpfer, O.P. Pavlenko, and G. Soff, Phys. Rev. D 54, 2399 (1996).

[3] P. Lévai and U. Heinz, Phys. Rev. C 57, 1879 (1998).

[4] J. I. Kapusta, Finite-Temperature Field Theory (Cambridge University Press, Cambridge, England, 1989).

[5] B. Kastening, Phys. Rev. D 56, 8107 (1997); T. Hatsuda, ibid. 56, 8111 (1997).

[6] F. Karsch, A. Patkós, and P. Petreczky, Phys. Lett. B 401, 69 (1997).

[7] I. T. Drummond, R. R. Horgan, P. V. Landshoff, and A. Rebhan, Nucl. Phys. B524, 579 (1998).

[8] J. M. Luttinger and J.C. Ward, Phys. Rev. 118, 1417 (1960).

[9] G. Baym, Phys. Rev. 127, 1391 (1962).

[10] E. Riedel, Z. Phys. 210, 403 (1968).

[11] B. Vanderheyden and G. Baym, J. Stat. Phys. 93, 843 (1998).

[12] J.-P. Blaizot, E. Iancu, and A. Rebhan (to be published).

[13] L. Dolan and R. Jackiw, Phys. Rev. D 9, 3320 (1974).

[14] E. Braaten and R. D. Pisarski, Nucl. Phys. B337, 569 (1990); J. Frenkel and J. C. Taylor, Nucl. Phys. B334, 199 (1990).

[15] U. Kraemmer, M. Kreuzer, and A. Rebhan, Ann. Phys. (N.Y.) 201, 223 (1990).

[16] O. K. Kalashnikov and V. V. Klimov, Sov. J. Nucl. Phys. 33, 443 (1981); H. A. Weldon, Phys. Rev. D 26, 1394 (1982).

[17] R. D. Pisarski, Physica (Amsterdam) 158A, 246 (1989).

[18] G. Boyd et al., Nucl. Phys. B469, 419 (1996).

[19] J. O. Andersen, E. Braaten, and M. Strickland, hep-ph/ 9902327; hep-ph/9905337.

[20] H. Schulz, Nucl. Phys. B413, 353 (1994).

[21] A. K. Rebhan, Phys. Rev. D 48, R3967 (1993).

[22] K. Kajantie et al., Phys. Rev. Lett. 79, 3130 (1997); M. Laine and O. Philipsen, hep-lat/9905004. 